\title{
DYNAMIC PERFORMANCE CHARACTERISTICS OF A THERMOELECTRIC GENERATOR
}

\author{
Ahmed S. El-Adl ${ }^{1, *}$, M.G. Mousa ${ }^{2}$, E.A. Abdel-Hadi ${ }^{3}$ and A.A. Hegazi ${ }^{2}$
}

\begin{abstract}
A thermoelectric generator (TEG) is a device that transforms thermal energy directly into electrical power by exploiting the Seebeck effect. In the current study, the dynamic performance characteristics of a TEG is experimentally studied under different operating conditions. The Influence of input heat rate and the influence of utilizing extended surfaces (fins) on both transient and steady-state performance of a TEG are experimentally investigated. The variation in the temperatures of the TEG hot-and cold-side in addition to the output voltage is taken as a denotation of the performance characteristics. Input heating rate of $15.0 \mathrm{~W}, 17.5 \mathrm{~W}, 20.0 \mathrm{~W}, 22.0 \mathrm{~W}$ and $25.0 \mathrm{~W}$ are applied to the TEG hot-side. Free air convection (FC) is utilized for heat dissipation from the TEG module through the cold-side. From the experimentation, it can be concluded that increasing the input heating rate provides a higher temperature difference between the module sides leading to higher power output. Additionally, using fins to aid heat dissipations improved the TEG performance by lowering the temperature of the cold-side and increasing the temperature difference across the module. The experimental data collected are compared with the data obtainable by the TEG module manufacturer and an excellent concordat is acquired.
\end{abstract}

Keywords: Thermoelectric Generator (TEG), Seebeck Effect, Fins, Temperature of the TEG Sides, Power Output, Conversion Efficiency, Transient and Steady-State Performance

\section{INTRODUCTION}

The accelerating imbalance between demand and provision supply of energy and continuous energy abjection motivates the researchers to investigate environmental affable, clean energy resources. Thermoelectric (TE) devices are among the most promoting and cost-effective energy resources. TEG provides versatile vantages over conventional electrical energy generators. TEGs are durable solid-state energy converters that have no mechanical moving parts and therefore are noiseless, lightweight, durable, dependable, compact, prolonged running periods, maintenance-free, environmentally friendly and uncomplicated scalability. The use of TEGs has increased fast in contemporary years with applications ranging from microwatts to kilowatts. From the most remarkable applications is the recovery of automotive waste heat [1], besides the recovery of waste heat from industrial processes [2]. Additionally, TEGs can be exploited to generate electric energy by direct conversion of renewable energy such as solar power [3]. Moreover, attributable to their robustness, TEGs are suitable for specialized applications such as medical, aerospace, telecommunications [4] and military. A typical TEG module consists of a number of semiconductor pairs. Each pair consists of a "p-type" (hole carriers) and an "n-type" (electron carriers) thermoelements (i.e., pellet) connected through metallic electrical contact pads, this pair is known as a thermoelectric couple [5]. A Schematic diagram of a single TE couple, which is illustrated in Figure 1.

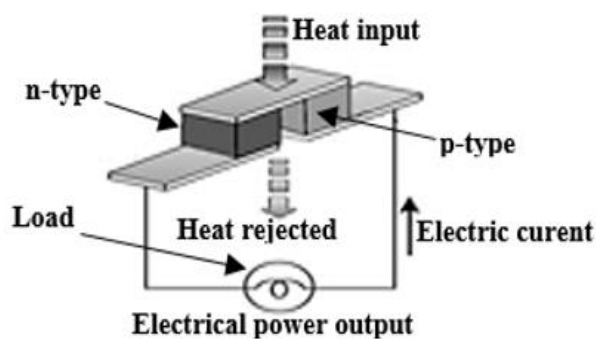

Figure 1. Schematic diagram of a single thermoelectric couple (Thermocouple) [6]

This paper was recommended for publication in revised form by Regional Editor Mohamed Awad

${ }^{1}$ Department of Mechanical Engineering, Higher Technological Institute, Tenth of Ramadan City, Egypt

${ }^{2}$ Department of Mechanical Power Engineering, Mansoura University, Mansoura, Egypt.

${ }^{3}$ Department of Mechanical Engineering, Benha University, Shoubra, Egypt.

*E-mail address: ahmed.s.eladl@htiedu.eg

Orcid id: https://orcid.org/0000-0001-7123-4785

Manuscript Received 10 November 2017, Accepted 02 February 2018 
In the module, the TE couples are connected electrically in series using a metal connecting strips and thermal in parallel, inserted between two thermally conducting and electrically insulating ceramic plates. A cutaway view of a conventional TEG module is illustrated in Figure 2.

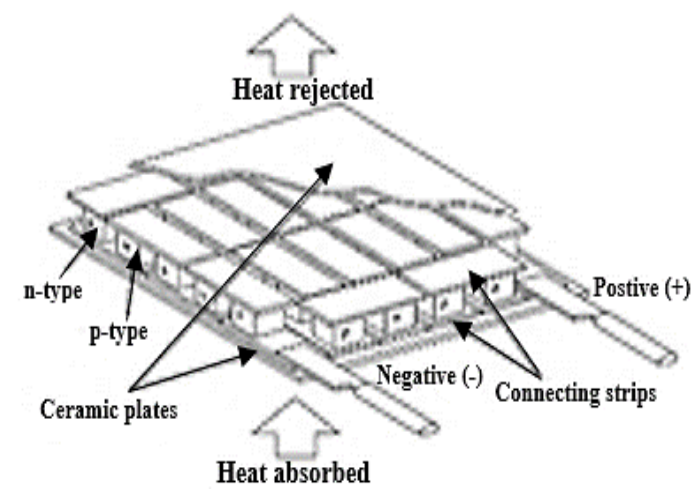

Figure 2. Cut-away view of a typical TEG module [6].

The conversion efficiency of TE materials highly relays on the figure of merit, $\mathrm{ZT}=\frac{\mathrm{s}^{2} \sigma}{\mathrm{k}} \mathrm{T}$, where " $\mathrm{S}$ " is the Seebeck coefficient, " $\sigma$ " is the electric conductivity, " $k$ " is the thermal conductivity, and " $T$ " is the absolute temperature at which the properties are evaluated. [7]. TE materials with high ZT values have been developed continuously $[8,9]$. In addition to exploring TE materials properties, improving the design of the TE module and the geometrical shape have been counted constantly [10,11].

Considerable research studies were also experimentally carried out to examine TE power generation and device efficiency. Sarhadi et al. [12] presented the influence of using different interface materials on powerproducing capabilities of a TEG as a function of flow rate. Three types of thermal interface materials (TIMs) were assessed. The optimal TIM was shown to be graphite paper. Wang et al. [13] experimentally investigated the influence of metal foams on the efficacy of TE waste heat recovery system. The obtained data illustrated that filling metal foams in the flow channels efficiently enhanced the performance of the TEG. A comparison between the thermal performances of TE generators cooled by either bare plate, $60 \mathrm{~mm}$ fin, $80 \mathrm{~mm}$ fin and $100 \mathrm{~mm}$ fin is presented by Date et al. [14]. It was concluded that the varying the fin length from $60 \mathrm{~mm}$ to $100 \mathrm{~mm}$ did not have as much influence on the cooling capacity and likewise did not provide as much effect on power generation from the TE generators. Montecucco et al. [15] analyzed the effectiveness of thermal imbalance on the power output from arrays of TE generators connected in parallel and in series. The obtained results suggested that the parallel electrical connection enabled less of the obtainable available power to be possessed. Lesage et al. [16] investigated TE power boosted of a liquid-to-liquid TEG. Three different geometric forms of flow turbulating inserts were integrated into the channels of the TE generator. Spiral inserts provided the minimal amelioration in TE power generation whilst inserts with protruding panels were the most effective. Rezania et al. [17] studied the coolant pumping power versus the power obtained from the TEG. The obtained data showed that there was a specific coolant flow rate at any temperature difference to provide maximum net-power from the system.

Concluding from the literature, there is a considerable thrust towards the enhancement of the output power of TEGs by boosting their performance in order to make them more competitive energy technology. In the present study, the impact of different operating conditions on transient and steady-state performance characteristics of a TEG is experimentally investigated. Operating aspects of the TEG system such as hot-side temperature, cold-side temperature, closed circuit voltage and power output are studied. The influence of heating rates through the TEG hot-side besides the employment of finned heat sink (fins) to aid heat dissipation from the module cold-side on the TEG performance is experimentally demonstrated. 


\section{EXPERIMENTAL SETUP AND PROCEDURES}

The experimental setup is designed and constructed to study the performance characteristics of the TEG for both transient and steady-states is shown in Figure 3. A thermoelectric Bismuth Telluride $\left(\mathrm{Bi}_{2} \mathrm{Te}_{3}\right)$ device designed for electric power generation, with a physical size of $62 \mathrm{~mm} \times 62 \mathrm{~mm} \times 4.0 \mathrm{~mm}$, containing $127 \mathrm{p}$-n couples connected in series has been used in this study. To supply the indispensable thermal input to TEG, the hotside is united to a copper plate, heated with a nominal power of $277 \mathrm{~W}$. The rate of the input heat is regulated using an electrical autotransformer (VARIAC) connected to a voltage stabilizer to ensure constant input heating rate to the TEG. An assembly is created to house the TEG for the testing purposes. The assembly consists of two copper plates; one is positioned between the hot-side of the module and the heater, while the other plate is positioned between the cold-side and the heat sink (fins). These copper plates are chosen for construction due to its high thermal conductivity, so heat is well distributed and thus temperature measurements of both sides more convenient. The copper plates are insulated by Phenolic Laminated sheets (for electric and thermal insulation), where groves are made in these sheets to occupy the copper plates. The lateral area of the test section is heavily insulated with a dense layer of glass wool (excluded from Figure 3) to prevent thermal forfeiture from the setup to the surrounding. To ensure proper thermal contact and minimum contact resistance, optimum-clamping force (i.e. as provided by module manufacturer) is applied on the TEG using screws and nuts. Additionally, a layer of TIM is placed between the copper plates and both sides of the TEG. The particular TIM used in the relevant setup is a thin graphite sheet having a thermal conductivity of $1600 \mathrm{~W} / \mathrm{m} \cdot \mathrm{K}$ and thickness of $0.025 \mathrm{~mm}$. Both hot- and coldside temperatures are measured using ten Chromel (Nickel-Chromium Alloy) / Alumel (Nickel-Aluminum Alloy) thermocouples (type $\mathrm{K}$ ) with $0.2 \mathrm{~mm}$ wire diameter, where five thermocouples are used for each side. The two terminals of the TEG are connected with a non-inductive power resistor $(0.47 \Omega)$ to form an electric circuit from which the output voltage and power output could be accurately measured. The temperature of module sides in addition to the output voltage is recorded using a data acquisition system model NI PCI-6013, manufactured by National Instruments Co. Also, all the thermocouples are connected to a temperature recorder through a multiswitch arrangement. The uncertainty in temperature measurements was $\pm 1.5^{\circ} \mathrm{C}$. The utilized fins consist of 15 fins with $2 \mathrm{~mm}$ thickness and $16 \mathrm{~mm}$ height. The gap between the fins is $2 \mathrm{~mm}$. Also, a layer of TIM is applied between the upper surface of the cold-side copper plate and the finned heat sink and to assure adequate contact between them. The fin is fixed over the copper plate through the usage of a pin - screw fixture threaded through two holes machined in the upper Phenolic Laminated sheet. The surrounding temperature is maintained at $30^{\circ} \mathrm{C}$ during all experiments.

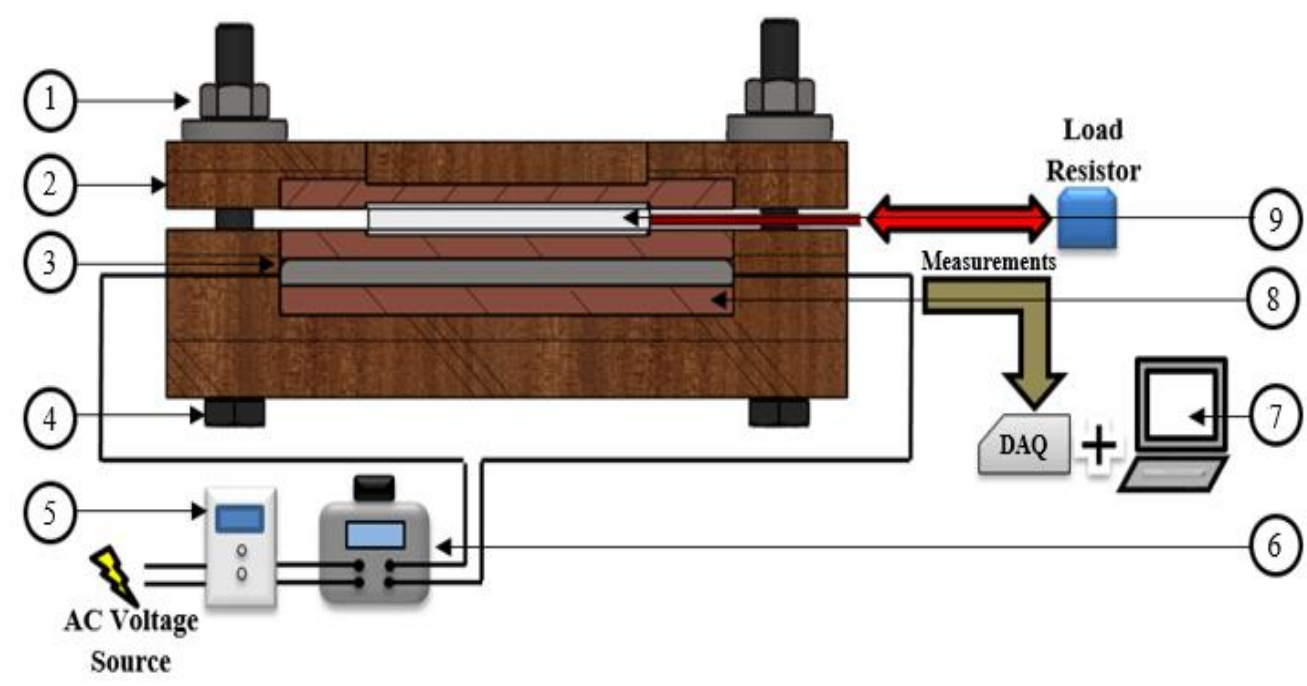

1. Nut, 2. Phenolic Laminated sheet, 3. Electric heater, 4. Screw 5. Voltage stabilizer, 6. Autotransformer (VARIAC), 7. Data acquisition system, 8. Copper plate, 9. TEG module.

Figure 3. Schematic diagram of the experimental setup. 


\section{DATA REDUCTION}

The experimental data acquired that include the hot- and cold-side temperatures beside the output voltage are used to calculate the power output and conversion efficiency. At steady-state condition, the generated heat by the electric heater can be calculated using the equation:

$$
\mathrm{Q}_{\text {in }}=\left(\frac{\mathrm{V}_{\mathrm{EH}}^{2}}{\mathrm{R}_{\mathrm{EH}}}\right)
$$

, where "Qin" is the heating rate provided by an electric heater $(\mathrm{W})$, " $\mathrm{V}_{\mathrm{EH}}$ " is the supplied voltage through the terminals of the electric heater $(\mathrm{V})$ and $\mathrm{R}_{\mathrm{EH}}$ is the resistance of the electric heater $(\Omega)$.

The power output from the TEG module is calculated by employing from the following equation:

$$
\mathrm{P}_{\mathrm{o}}=\left(\frac{\mathrm{V}_{\mathrm{o}}{ }^{2}}{\mathrm{R}_{\mathrm{L}}}\right)
$$

, where "Po" is the power output $(\mathrm{W})$, " $\mathrm{V}_{\mathrm{o}}$ " is the output voltage $(\mathrm{V})$ and " $\mathrm{R}_{\mathrm{L}}$ " is the load resistor $(\Omega)$.

By calculating the power output of the TEG module to the connected resistive load, the conversion efficiency can be obtained by using the following equation:

$$
\eta_{\mathrm{c}}=\frac{\mathrm{P}_{\mathrm{o}}}{\mathrm{Q}_{\text {in }}}
$$

where " $\eta_{\mathrm{c}}$ " is the conversion efficiency (\%).

\section{RESULT AND DISCUSSION}

In experimental results, the effect of different operating conditions on both transient and steady-state performance of the TEG module is presented. A comparison between the current experimental results of the variation in the power output with hot-side temperature and those provided by the manufacturer [18] in case of FC aided by finned heat sink is illustrated in Figure 4. From this figure, it can be noticed that a stellar accord between both results, illustrating that the power output increases almost linearly with increasing the hot-side temperature. The stratification between the experimental data of the current study and those provided by the manufacturer of the TEG module illustrates the veracity of the procedures of the carried-on experiments and the accuracy of the measurement. The tenuous inconsistency (i.e. within $2.5 \%$ ) both results returns to the diversity between the utilized measurement methods in the current study and that of employed by the manufacturer.

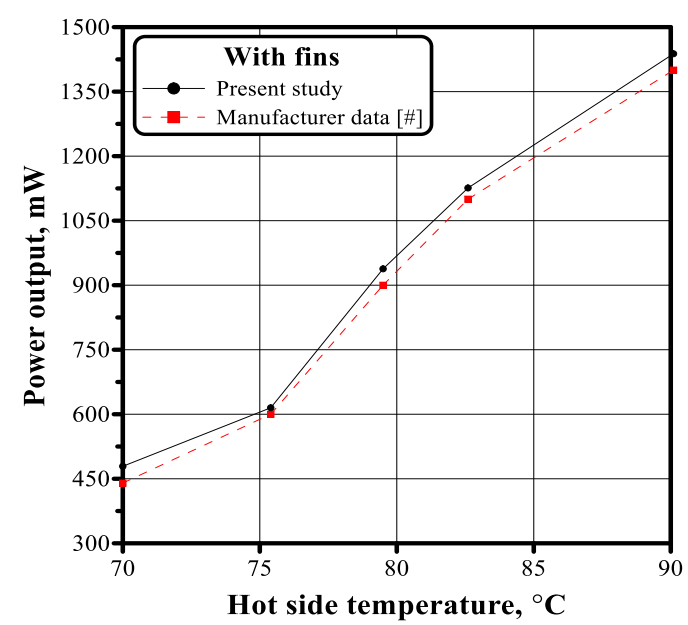

Figure 4. Comparison between the experimental results of the current study and that after reference [18], for the variation in the power output with hot-side temperature. 
The Influence of heating rate on the variation of both sides temperature with time in case of FC is illustrated in Figure 5. Figures 5a-5b show the variation of the temperature with time for the hot- and cold-side without and with fins for various values of input heating rate, respectively.

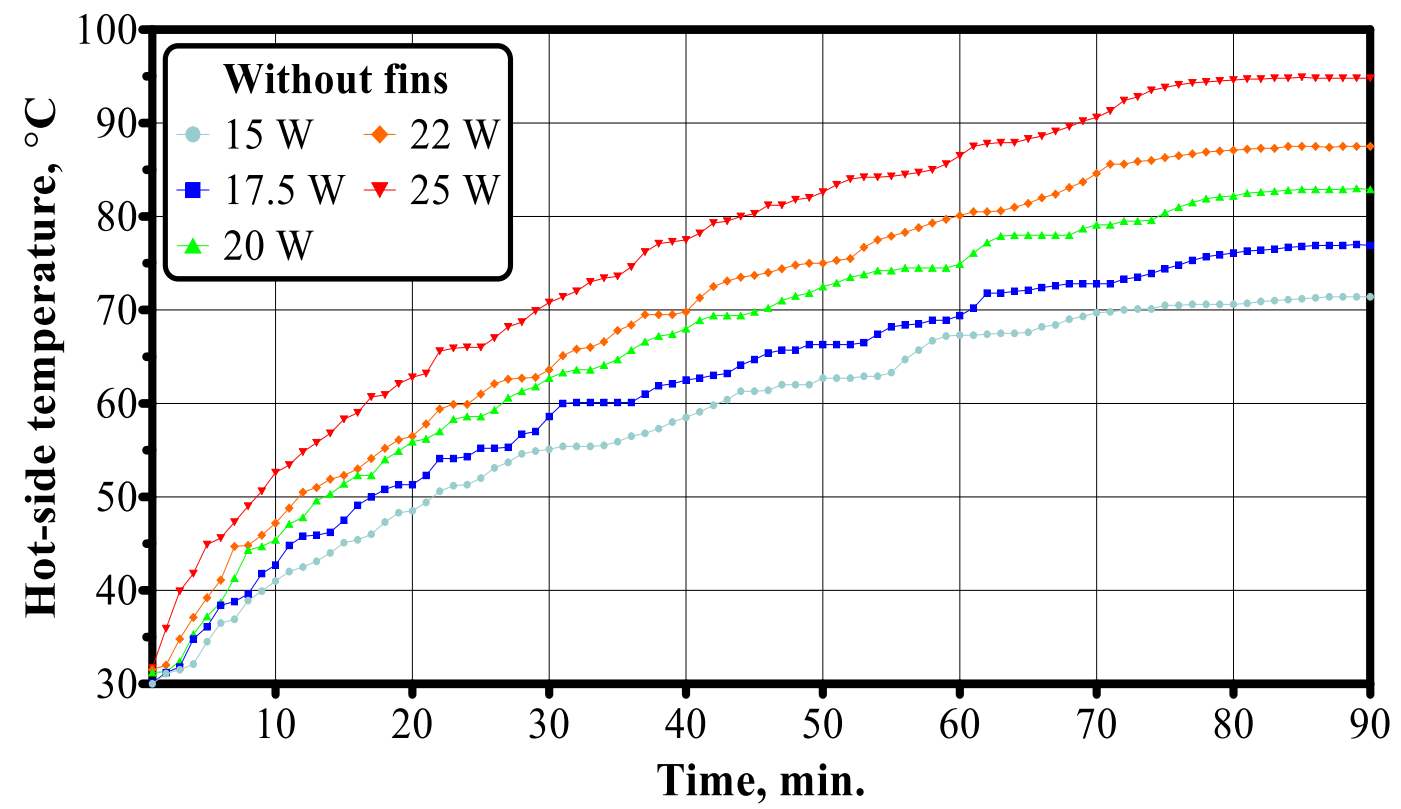

(a)

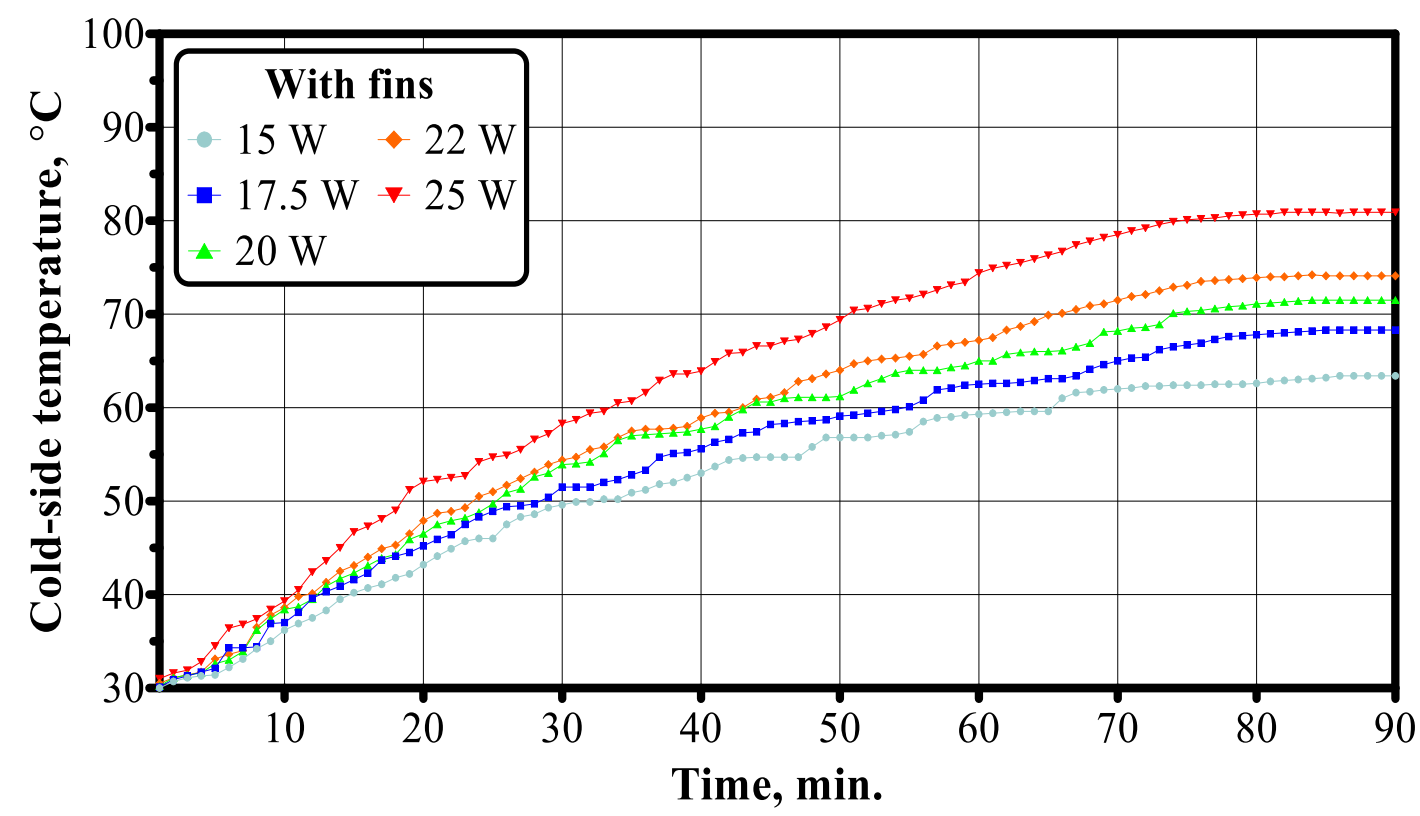

(b)

Figure 5. Influence of heating rate on the variation in the temperature of both sides with time, in case of FC (a) Variation in the hot-side temperature with time for various input heating rates, in case of FC without the assist of fins. (b) Variation in the cold-side temperature with time for various input heating rates, in case of FC with the assist of fins

It can be noticed from these preceding figures that for all the operating conditions in the experimental studies, the temperature of both sides increases by increasing the heating rate, whereas the system heats up relatively quickly in the beginning and then slowly converts to steady-state operation. 
Additionally, by comparing the temperatures of both sides for all operating conditions studied, it can be deduced that the temperature of the cold-side is always lower than that of the hot-side. This is related to the cooling Influence provided by heat dissipation.

In addition, it can be noticed that the temperature of both sides follows the same trend of transitory increase with operating time and until a steady-state is achieved. This occurrence is caused by the direct Influence of heat conduction through the module from the hot-side to the cold-side. Therefore, the thermal conductivity of an excellent TE material must be comparatively as low as possible for a high-temperature difference could be obtained and maintained across the module sides. It is worthy to note that in addition to the effect of heat conduction (i.e., Fourier's heat), the temperature of both sides is also affected by the Peltier effect, Joule heating and Thomson effect, which occur when a current flow through the module due to the connection of a load to the TEG output terminals.

The Influence of heating rate on the variation in the output voltage with time, in the case of FC, is shown in Figure 6. Figures $6 \mathrm{a}-6 \mathrm{~b}$ demonstrate the variation of output voltage with time, in case of FC without and with the assist of fins, respectively for various values of input heating rate.
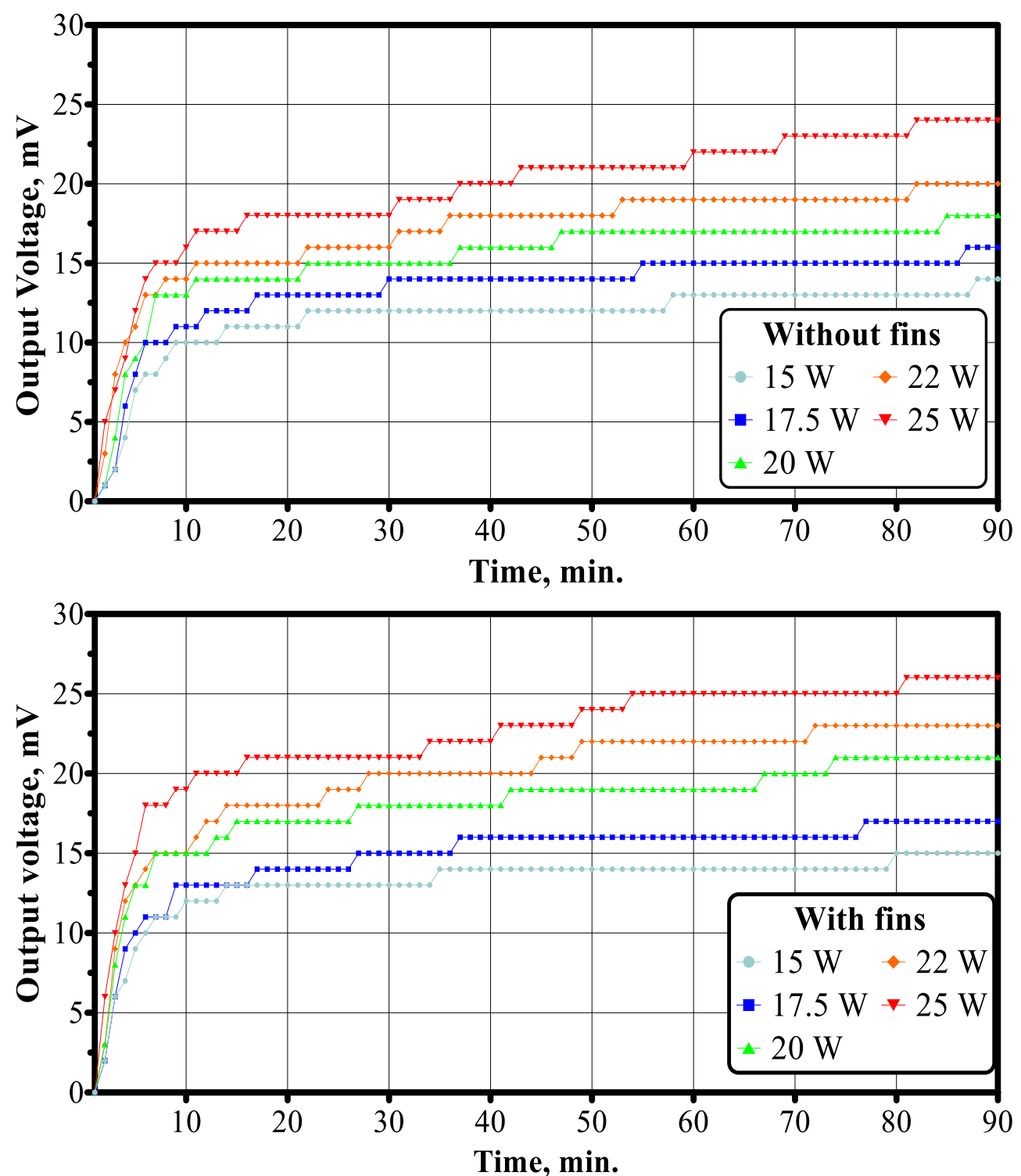

Figure 6. Influence of heating rate on the variation in the output voltage with time, in case of FC (a) Variation in the output voltage with time for various input heating rates, in case of FC without the assist of fins, (b) Variation in the voltage output with time for various input heating rates, in case of $\mathrm{FC}$ with the assist of fins 
According to the Seebeck Influence, TEG generates a voltage when a temperature difference is instituted between its sides. The increased inward heating rate at the TEG hot-side permits for a much higher temperature difference across the module and therefore increased output voltage generation. This, in turn, improves the power output, which can be noticed from the figures mentioned above. At the start of TEG operation, the temperature difference across the two sides of the module is zero that accordingly will lead to zero output voltage to be generated from the TEG (i.e., zero power output). With the transient time increasing, the temperature difference between the sides of the module increases with step trend due to quasi-steady thermal response and until the steadystate operation is achieved. Additionally, it can be observed from Figures 4 and 5 that the output voltage and the temperature difference between both sides are almost synchronously achieved stability. Steady-state performance is used to summarize and clarify the obtained results.

The Influence of heating rate on the temperature of TEG for both the hot- and cold-side is illustrated in Figure 7. Figures $7 \mathrm{a}-7 \mathrm{~b}$ show the variation in the temperature of both sides with input heating rates, in case of FC without and with the assist of fins, respectively. Generally, it can be noticed from the figures that the temperature of both sides varies almost linearly with the input heating rate. Where the temperatures of the hot- and cold-side are directly proportional to the value of heating rate and the temperature of the cold-side is intuitively lower than the temperature of the cold-side due to the influence of heat dissipation causing the temperature difference between both sides.

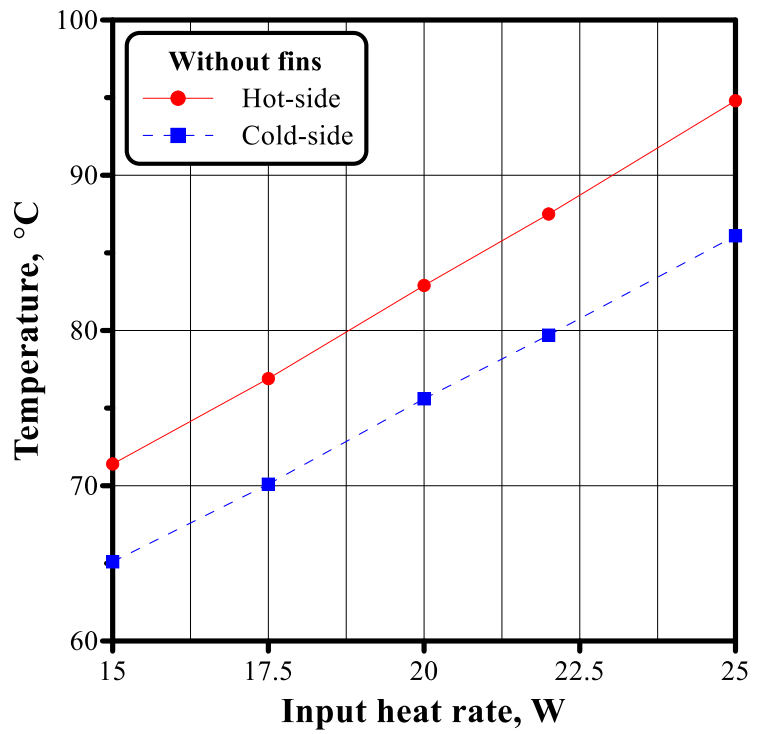

(a)

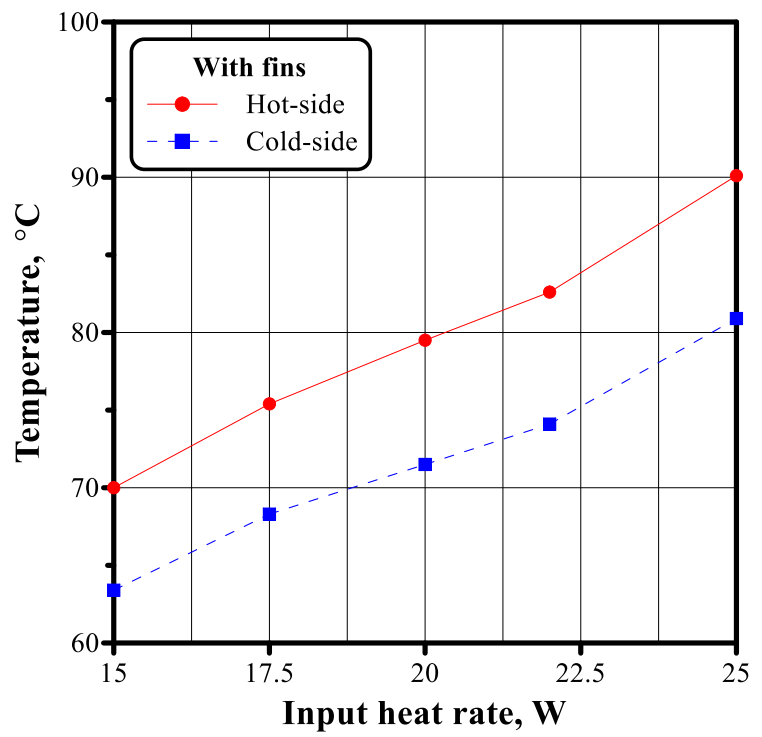

(b)

Figure 7. Influence of input heating rate on the temperature of both sides (a) Variation in the temperature of both sides with input heating rate, without fins, (b) Variation in the temperature of both sides with input heating rate, with fins

The Influence of heating rate on the output voltage and the corresponding power output of TEG is shown in Figure 8. Figures $8 \mathrm{a}-8 \mathrm{~b}$ show the variation in the output voltage and the corresponding power output in case of FC without and with the assist of fins for various heating rates, respectively. It can be illustrated that the output voltage and consequently the power output is directly proportional to the value of input heat. Increasing the input heating rate will stimulate the temperature difference between the sides of the module, by increasing the temperature of the hot-side that in turn causes a higher output voltage and subsequently enhanced power output. Additionally, it can be noticed that both output voltage and power output follow the exact trend for all studied cases. This intuitively returns to the dependency of power output on the output voltage. 


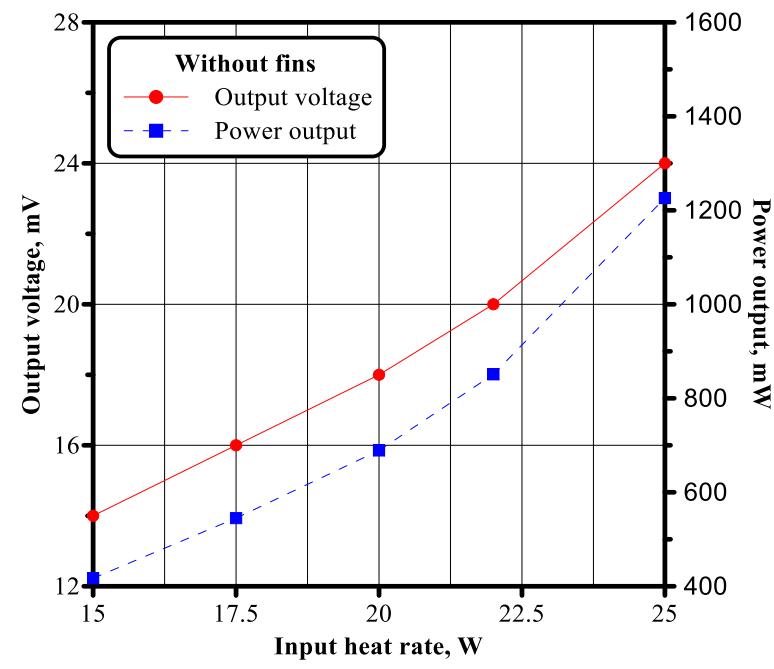

(a)

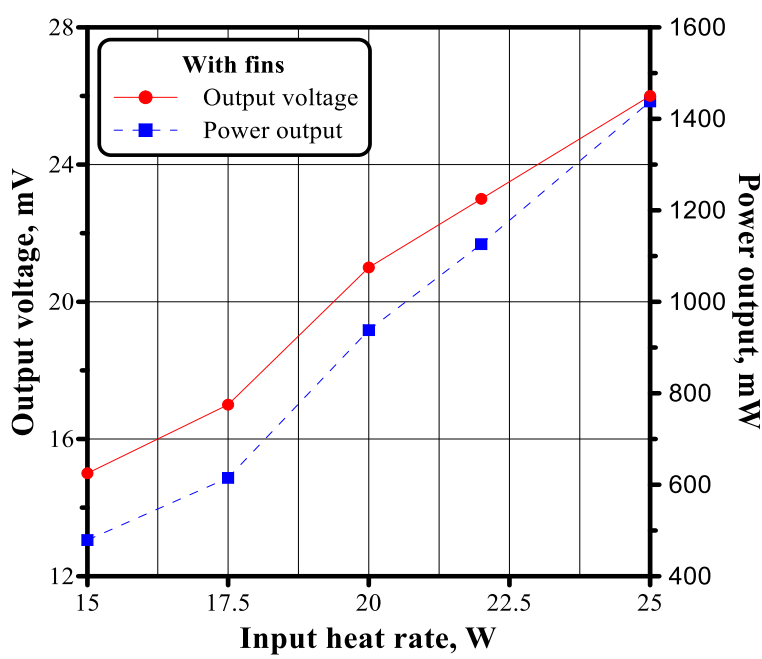

(b)

Figure 8. Influence of input heating rate on the output voltage and power output (a) Variation in the output voltage and power output with input heating rate, without fins, (b) Variation in the output voltage and power output with input heating rate, with fins

The Influence of employing fins to assist heat dissipation from the module cold-side on the temperatures of the TEG hot- and cold-side is shown in Figure 9. It can be noticed that using fins with the TEG lowers the temperature of both TEG sides by augmenting the rate of heat transfer from the module cold-side promoted by increasing the convective heat transfer area subjected to surrounding heat sink. This causes a higher temperature difference between the TEG sides with an average increase of $4.8 \%$ up to $5.7 \%$.

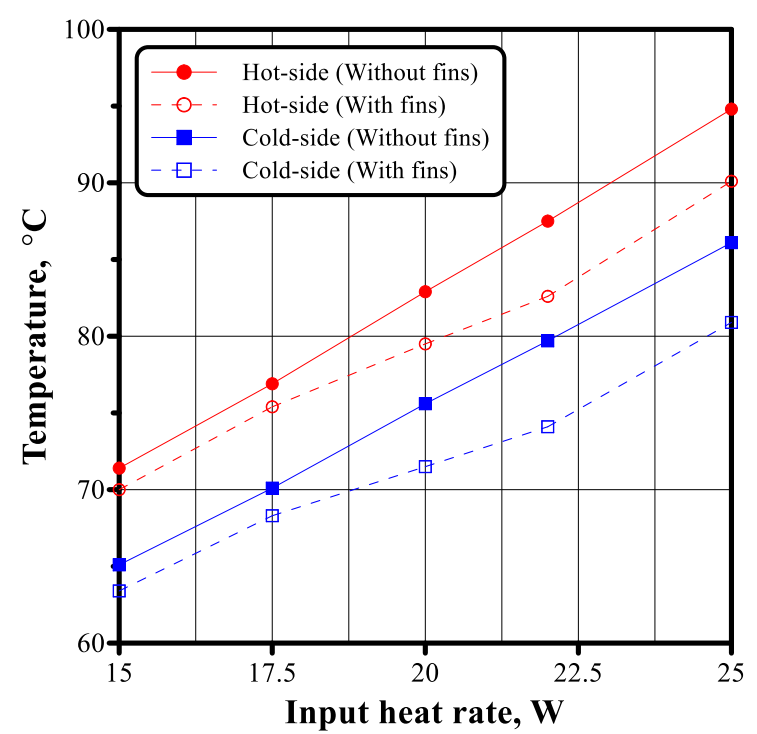

Figure 9. Influence of using fins on the temperature of both sides

The Influence of employing fins to assist heat dissipation from the module cold-side on the output voltage and power output is shown in Figure 10. Figures 10a-10b show the variation in the output voltage and the corresponding power output for various heating rates, respectively. Since the employment of fins to assist heat transfer from the TEG causes higher temperature difference across the TEG sides it will subsequently improve the output voltage, and in turn, the power output is enhanced with an average increase of $14.9 \%$ to $17.3 \%$. 


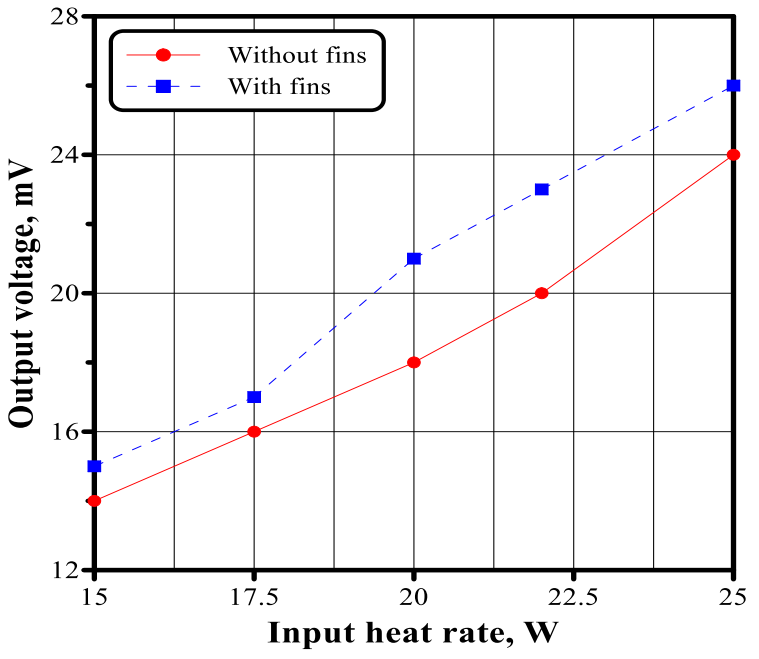

(a)

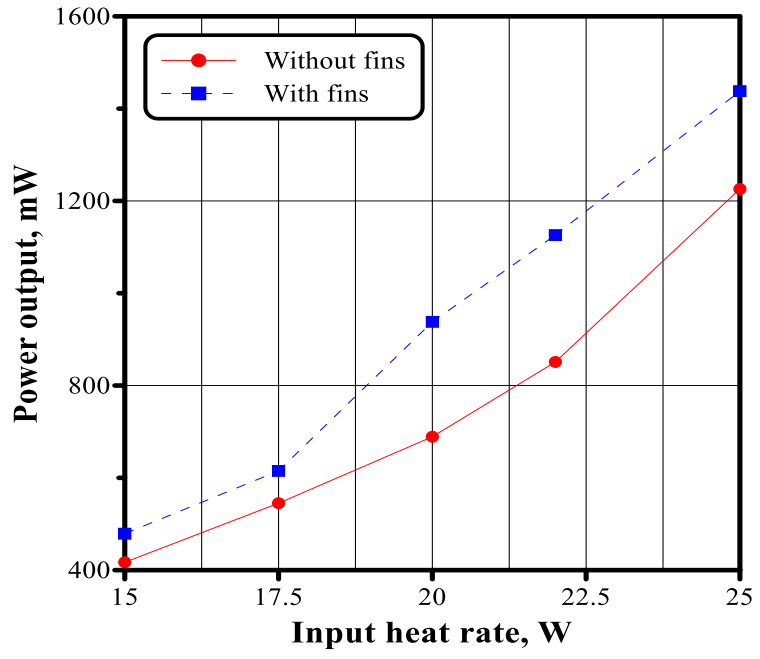

(b)

Figure 10. Influence of using fins on the output voltage and power output (a) Variation in the output voltage with input heating rate, (b) Variation in the power output with input heating rate

The Influence of employing fins to assist heat dissipation from the module cold-side on the conversion efficiency is shown in Figure 11. It can be concluded that the increasing heating rate will motivate the TEG to generate more output voltage that consequently will increase the power output and promotes, in turn, the acquisition of higher conversion efficiency. Since the conversion efficiency is defined as the ratio between the power output from the TEG to the rate of heat input, it can be expected that the enhancing effect caused by the utilization of fins on power output for the same input heating rate will consequently improve the conversion efficiency causing an average increase of $14.3 \%$ up to $18.4 \%$.

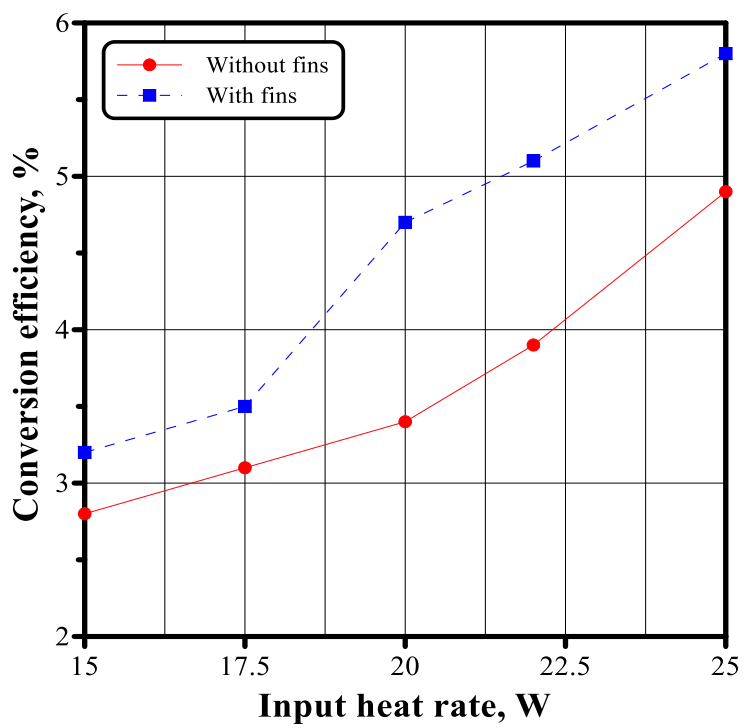

Figure 11. Influence of using fins on the conversion efficiency

\section{CONCLUSION}

In the present study, an experiential investigation on the transient and steady-state performance characteristics of a TEG is carried out. The influence of varying the input heating rate to the TEG hot-side besides the influence of employing fins to aid the heat dissipation by free air convection from the TEG cold-side on the performance is enquired. Based on the acquired results of the current study, it can be noticed that increasing the input heating rate causes an increase in both the hot- and the cold-side temperatures as well as increasing the temperature difference. Hence, according to the Seebeck effect, the output voltage increases, leading to an increase 
in the power output and accordingly increases the conversion efficiency. In addition, it was observed that utilizing fins to assist heat rejection from the TEG causes an average increase of $14.9 \%$ up to $17.3 \%$ in power output. This is attributed to the reduction in the temperature of cold-side, which leads to a higher temperature difference between the module sides, improves the generated output voltage, consequently increases the power output and enhances the conversion efficiency. An average increase of $14.9 \%$ up to $17.3 \%$ in conversion efficiency is achieved by using fins to aid heat rejection from the TEG. A maximum conversion efficiency achieved was $5.8 \%$ in case of input heating rate of $25.0 \mathrm{~W}$ and with employing the fins to aid heat dissipation from the module cold-side.

$\begin{array}{ll}\text { Nomenclature } \\ \mathrm{Bi}_{2} \mathrm{Te}_{3} & \text { Bismuth Telluride } \\ F C & \text { Free convection } \\ k & \text { Thermal Conductivity, } \mathrm{W} / \mathrm{m} \cdot \mathrm{K} \\ n & \text { Negative } \\ p & \text { Positive } \\ P & \text { Power, W } \\ Q & \text { Heating rate, W } \\ R & \text { Electric resistance, } \Omega \\ S & \text { Seebeck coefficient, } \mathrm{V} / \mathrm{K} \\ T & \text { Absolute temperature, } \mathrm{K} \\ T E & \text { Thermoelectric } \\ T E G & \text { Thermoelectric generator } \\ T I M & \text { Thermal interface material } \\ V & \text { Voltage, } \mathrm{V} \\ Z T & \text { Figure of merit } \\ \text { Greek symbols } \\ \Delta & \text { Difference } \\ \eta & \text { Efficiency, } \% \\ \Omega & \text { Ohm } \\ \sigma & \text { Electric conductivity, } \mathrm{S} \\ \text { Subscripts } & \\ c & \text { Conversion } \\ E H & \text { Electric heater } \\ i n & \text { Input } \\ L & \text { Ooad } \\ O & \\ & \end{array}$

\section{REFERENCES}

[1] Kim, T., Negash, A., and Cho, G. (2016). Waste heat recovery of a diesel engine using a thermoelectric generator equipped with customized thermoelectric modules. Energy Conversion and Management, 124, 280-286.

[2] Ding, L., Meyerheinrich, N., Tan, L., Rahaoui, K., and Jain, R., Akbarzadeh A. (2017). Thermoelectric power generation from waste heat of natural gas water heater. Energy Procedia, 110, 32-37.

[3] Shafii, M., Shahmohamadi, M, Faegh, M., and Sadrhosseini, H. (2016). Examination of a novel solar still equipped with evacuated tube collectors and thermoelectric modules. Desalination, 382, 21-27.

[4] BOBEAN, C. and Valentina, P. (2013). The Study and Modeling of a Thermoelectric Generator Module, The 8th international symposium on advanced topics in electrical engineering.

[5] Jang, J. and Tsai, Y. (2013). Optimization of thermoelectric generator module spacing and spreader thickness used in a waste heat recovery system, Applied Thermal Engineering, 51, 677-689.

[6] HoSung, L., (2011). Thermal Design: Heat Sinks, Thermoelectrics, Heat Pipes, Compact Heat Exchangers, and Solar Cells. JOHN WILEY \& SONS, INC., New York. 
[7] Barma, M., Riaz, M., Saidur, R. and Long, B. (2015) Estimation of thermoelectric power generation by recovering waste heat from Biomass fired thermal oil heater, Energy Conversion and Management, 98, 303-313.

[8] Du, H., Su, T., Li, H., Li, S., Hu, M., Liu, B., Ma, H. and Jia, X. (2016). Enhanced low temperature thermoelectric performance and weakly temperature-dependent figure-of-merit values of $\mathrm{PbTeePbSe}$ solid solutions. Journal of Alloys and Compounds, 658, 885-890.

[9] Li, J., Li, D., Qin, X. and Zhang, J. (2017). Enhanced thermoelectric performance of p-type SnSe doped with Zn. Scripta Materialia, 126, 6-10.

[10] Cao, Z., Koukharenko, E., Tudor, M., Torah R and Beeby, S. (2016). Flexible screen printed thermoelectric generator with enhanced processes and materials. Sensors and Actuators, A238, 196-206.

[11] Huang, K., Li, B., Yan, Y., Li, Y., Twaha, S. and Zhu J. (2017). A comprehensive study on a novel concentric cylindrical thermoelectric power generation system. Applied Thermal Engineering, 117, 501-510.

[12] Sarhadi, A., Bjørk, R., Lindeburg, N., Viereck, P. and Pryds, N. (2016). A thermoelectric power generating heat exchanger: Part II - Numerical modeling and optimization. Energy Conversion and Management, 119, 481487.

[13] Wang, T., Luan, W., Liu, T., Tu, S. and Yan, J. (2016). Performance enhancement of thermoelectric waste heat recovery system by using metal foam inserts. Energy Conversion and Management, 124, 13-19

[14] Date, A., Date, A., Dixon, C., Singh, R. and Akbarzadeh, A. (2015). Theoretical and experimental estimation of limiting input heat flux for thermoelectric power generators with passive cooling. Solar Energy, 111, 201-217.

[15] Montecucco, A., Siviter, J. and Knox, A. (2014). The effect of temperature mismatch on thermoelectric generators electrically connected in series and parallel, Applied Energy, 123, 47-54

[16] Lesage, F., Sempels, É. and Lalande-Bertrand, N. (2013). A study on heat transfer enhancement using flow channel inserts for thermoelectric power generation, Energy Conversion and Management, 75, 532-541.

[17] Rezania, A., Rosendahl, L. and Andreasen, S. (2012). Experimental investigation of thermoelectric power generation versus coolant pumping power in a microchannel heat sink, International Communications in Heat and Mass Transfer, 39, 1054-1058.

[18] TEG (GM250-127-28-10) manufacturer datasheet, http://www.europeanthermodynamics.com. 